\title{
Generation of superoxide anion radicals and platelet glutathione peroxidase activity in patients with schizophrenia
}

\author{
This article was published in the following Dove Press journal: \\ Neuropsychiatric Disease and Treatment \\ 5 May 2014 \\ Number of times this article has been viewed
}

\section{Anna Dietrich-Muszalska Anna Kwiatkowska}

Department of Biological Psychiatry of the Chair of Experimental and Clinical Physiology, Medical University of Lodz, Lodz, Poland
Correspondence: Anna DietrichMuszalska

Department of Biological Psychiatry of the Chair of Experimental and Clinical Physiology, Medical University of Lodz, 6/8 Mazowiecka Street, Lodz 92-215, Poland

Email tzn_lodz@post.pl
Abstract: Blood platelets are considered to be a peripheral marker in schizophrenia and other psychiatric disorders. Oxidative stress in schizophrenia may be responsible for changes in platelet metabolism and function; therefore, the aim of this study was to examine and compare the generation of superoxide anions and activity of an antioxidant enzyme (glutathione peroxidase [GPx]) in blood platelets in patients with schizophrenia and healthy subjects. The level of superoxide anions generated in platelets after thrombin and platelet-activating factor stimulation and activity of GPx in patients with schizophrenia and healthy volunteers was estimated. The results obtained from the study indicate that the generation of superoxide anions in platelets as a response of platelets in patients with schizophrenia to such activating factors as thrombin or platelet-activating factor is higher than in the response of platelets of healthy subjects. In platelets from schizophrenic patients, suppressed GPx activity of about $67 \%$ was observed.

Keywords: schizophrenia, blood platelet, superoxide anion, activating factors, glutathione peroxidase

\section{Introduction}

Schizophrenia is one of the mental disorders in which the pathogenic mechanisms are complex and poorly understood. A growing body of evidence appears to implicate the role of oxidative stress with an impaired antioxidant defense system in this disease. $^{1-9}$ Molecular processes, including oxidative stress, that play a role in the etiopathology of schizophrenia cannot be studied directly in human brain tissue. Postmortem brain tissue has also been used extensively to study schizophrenia. ${ }^{1}$ For studies of oxidative stress in mental diseases, various cellular models are helpful (erythrocytes, lymphocytes, blood platelets, and cultured skin fibroblasts), as well as peripheral tissue models, since molecular processes associated with oxidative damage occur in all tissues. ${ }^{3,10}$ In the present study of oxidative stress in schizophrenic patients, blood platelets were used. Blood platelets are recognized as cellular models in neurobiological studies, because of their similarity to neurons, having the same embryological origin; platelets and neurons of the central nervous system show many similarities, including monoamine oxidase activity, serotonin reuptake, presence of neurotransmitter receptors, and exocytosis. ${ }^{11-14}$ Blood platelets are highly reactive, and within seconds of exposure to such agonists as thrombin or platelet-activating factor (PAF), they undergo activation, with a dramatic change in their morphology and biochemical events. PAF is a phospholipid mediator of inflammation with a wide range 
of biological activities, including alteration of the barrier function of endothelium. It is a strong platelet activator involved in migraine. ${ }^{15,16}$ Free radical-mediated pathological reactions (oxidative modification of biomolecules) may play a role in schizophrenia. A variety of different physiological agonists can activate platelets, and upon the activation of platelets, a cascade of morphological changes and biochemical reactions concomitant with the generation of free radicals and reactive oxygen species (ROS) takes place. ${ }^{17,18}$ ROS, including superoxide radicals $\left(\mathrm{O}_{2}^{-}\right)$, are generated during platelet activation induced by strong platelet agonists, and may be involved in platelet-signaling pathways. ${ }^{17-20}$ Under physiological conditions, superoxide radicals are constantly produced in the mitochondrial electron-transport chain. Platelets generate superoxide radicals mainly via the nicotinamide adenine dinucleotide phosphate (NADPH) oxidase or xanthine oxidase. The main source of superoxide radicals in activated platelets is the metabolism of arachidonate and the glutathione (GSH) cycle. ${ }^{17-20}$

The aim of the present study was to determine and compare the generation of superoxide radicals in resting as well as in thrombin- and PAF-stimulated platelets from patients with schizophrenia and healthy subjects. Polyunsaturated fatty acids (PUFAs; including arachidonic acid present in platelet-membrane phospholipids) are especially vulnerable to attack by ROS and the formation of lipid hydroperoxides, because of the high concentration of allylic hydrogen in their structure. Antioxidative enzymes, including GSH peroxidase (GPx), protect cells against the ROS-mediated changes. GPx converts peroxides and hydroxyl radicals into nontoxic forms, together with the concomitant oxidation with reduced GSH. Reduced GSH levels in cerebrospinal fluid and the prefrontal cortex in patients with schizophrenia have been described, ${ }^{21}$ and the deficit of GSH and its metabolite - $\gamma$-glutamylglutamine - in the cerebrospinal fluid of patients with schizophrenia who were drug-naïve or drug-free at that time was also found. ${ }^{22}$ Recently, the results of several studies have indicated the efficacy of $N$-acetylcysteine, a GSH precursor that is a useful agent in the treatment of various psychiatric disorders, including schizophrenia. ${ }^{23,24}$ The activity of GPx reducing lipid hydroperoxides in the platelets of schizophrenic patients is not well known; therefore, the purpose of the study was also to estimate the activity of this key antioxidant enzyme in platelets from patients with schizophrenia. There are conflicting data in the literature on the activities of antioxidant enzymes in patients with schizophrenia, measured mainly in erythrocytes. ${ }^{1,6,7}$

\section{Materials and methods Criteria for schizophrenic patient inclusion}

Thirty schizophrenic patients were included in the study: 20 male and ten female, aged 18-36 years, who were treated in the Department of Psychiatry of the Medical University of Lodz, Poland. All patients were interviewed and completed a special questionnaire on their treatment, course of disease, and extrapyramidal symptoms, measured by the SimpsonAngus Scale and Barnes Akathisia Scale.

The Mini-International Neuropsychiatric Interview for psychiatric examination was performed. ${ }^{25}$ All patients were diagnosed with paranoid schizophrenia, according to Diagnostic and Statistical Manual of Mental Disorders, fourth edition criteria ${ }^{26}$ (the acute period of psychosis), and they were treated with a stable dose of second-generation antipsychotic drugs (risperidone $4-6 \mathrm{mg} /$ day, quetiapine $600-750 \mathrm{mg} /$ day, and olanzapine $15-20 \mathrm{mg}$ /day; duration of treatment $2.0 \pm 1.5$ months). They did not use antidepressants or mood stabilizers. The mean duration of schizophrenia in the subjects was 4 years.

The control group consisted of 30 healthy subjects workers and students of the Medical University of Lodz - who were age- and sex-matched. Both groups belonged to the same community, and had similar nutritional habits. Both groups were on a balanced diet without antioxidant supplementation. Patients and control subjects with somatic illnesses were excluded. None of the control subjects or patients received aspirin or any other antiplatelet drugs, and none were alcohol/ drug abusers or showed clinical manifestation of allergy or infection. Heavy smokers were excluded from the group of patients. The majority of patients were not smokers (83\%), $17 \%$ of patients did not smoke in the week before blood drawing. There were no smokers in the control group. The body mass index of all subjects ranged around normal values. For each subject, blood-platelet counts were recorded, as well as medical history and medications used.

PAF, thrombin, and cytochrome $\mathrm{C}$ were purchased from Sigma-Aldrich (St Louis, MO, USA). All other reagents were of analytical grade, and were provided by commercial suppliers.

\section{Isolation of blood platelets}

Blood samples $(2 \times 9 \mathrm{~mL})$ from fasting schizophrenic patients and healthy volunteers were drawn between 8 and 8.30 am from a forearm vein into tubes containing acid citrate dextrose solution $(5: 1, \mathrm{v} / \mathrm{v})$. Platelets were isolated by differential centrifugation of fresh blood $\left(20\right.$ minutes, $\left.200 \mathrm{~g}, 20^{\circ} \mathrm{C}\right) .{ }^{27}$ 
Platelet-rich plasma was separated and centrifuged for 20 minutes at $1,000 \mathrm{~g}, 20^{\circ} \mathrm{C}$ to sediment platelets. The resulting platelet pellet was gently resuspended in modified Tyrode's buffer (140 mM NaCl, 10 mM glucose, 15 mM Tris/ $\mathrm{HCl}, \mathrm{pH} 7.4)$ in the presence of apyrase ( $1 \mathrm{mg} / \mathrm{mL})$, and the platelets were subsequently washed and then suspended in the same buffer. The entire washing procedure was performed in plastic tubes and carried out at room temperature. Blood platelets were suspended in modified Tyrode's buffer at a final concentration of $5 \times 10^{8}$ platelets $/ \mathrm{mL}$. Platelet suspensions were incubated with PAF $\left(1 \times 10^{-8} \mathrm{M}\right)$ or with thrombin $(0.4 \mathrm{U} / \mathrm{mL})$ for 2 minutes at $37^{\circ} \mathrm{C}$, and then the generation of superoxide radicals was determined. ${ }^{28}$ The platelets in the suspension were counted by the photometric method, as described by Walkowiak et al. ${ }^{29}$

The protocol was passed by the Committee for Research on Human Subjects of the Medical University of Lodz, number RNN/899/2000. All patients and volunteers included in the study were informed about the aims of the study and the methods implemented, and gave their written informed consent for participation in this study.

\section{Generation of superoxide anion radicals}

The generation of superoxide anions in control platelets (from healthy subjects) and in platelets from patients with schizophrenia (resting and PAF- or thrombin-stimulated platelets) was measured by means of superoxide dismutase $(1 \mu \mathrm{g} / \mu \mathrm{L})$-inhibitable reduction of cytochrome $\mathrm{C}$, as described by Jahn and Hänsch. ${ }^{28}$ Briefly, an equal volume of $\mathrm{Ca}^{2+} /$ $\mathrm{Mg}^{2+}$ free Tyrode buffer ( $\mathrm{pH} 7.4$ ), containing cytochrome $\mathrm{C}$ $(160 \mu \mathrm{mol} / \mathrm{L})$, was added to a $1 \mathrm{~mL}$ suspension of platelets. After 2 minutes of incubation, the platelets were sedimented by centrifugation ( $2000 \mathrm{~g}$ for 5 minutes), and the supernatants were transferred to cuvettes. The reduction of cytochrome $\mathrm{C}$ was measured spectrophotometrically at $550 \mathrm{~nm}$. The concentration of superoxide anions was evaluated on a basis of the molar extinction coefficient of reduced cytochrome $\mathrm{C}$ $\left(\varepsilon=18,700 \mathrm{M}^{-1} \cdot \mathrm{cm}^{-1}\right)$. All samples were in triplicate.

\section{Determination of glutathione peroxidase activity}

GPx activity was measured according to the spectrophotometric method of Paglia and Valentine. ${ }^{30}$ Briefly, the assay mixture consisted of $50 \mathrm{mM}$ Tris $\mathrm{HCl}, 0.1 \mathrm{mM}$ ethylenediaminetetraacetic acid, $\mathrm{pH} 7.6$ containing $2 \mathrm{mM} \mathrm{GSH}$, $0.14 \mathrm{mM}$ NADPH and $0.7 \mathrm{U} / \mathrm{mL}$ GSH reductase. Platelet suspensions were preincubated for 2 minutes at $37^{\circ} \mathrm{C}$ in the assay mixture, and the reaction was initiated by the addition of $0.2 \mathrm{mM}$ tert-butyl-hydroperoxide. Absorbance at $340 \mathrm{~nm}$ was recorded on a spectrophotometer. Results were expressed as nanomoles of NADPH oxidized per minute per milligram of platelet protein, using a molar extinction coefficient of $6.22 / \mathrm{cm}^{2} / \mu \mathrm{mol}$ for NADPH.

\section{Protein assay}

Protein concentration was determined according to Bradfort, using bovine serum albumin as a standard. ${ }^{31}$

\section{Statistical analysis}

Statistical analysis was done by several tests. The MannWhitney $U$ test was used for comparisons between the groups of healthy subjects and schizophrenic patients. For comparison of the generation of superoxide anion radicals after stimulation (thrombin or PAF) with resting platelets derived from schizophrenic patients or healthy subjects, the Wilcoxon signed-rank test was used. All values were expressed as means \pm standard error of the mean. $P$-values $<0.05$ were considered statistically significant.

\section{Results}

The results showed that in unstimulated resting platelets obtained from patients with schizophrenia, the level of generated superoxide anions was lower than in platelets derived from healthy subjects (Figure $1 \mathrm{~A}, P<0.05$ ). After thrombin stimulation, platelets from patients with schizophrenia generated higher amounts of superoxide anions than those from healthy subjects (Figure 1B, $P<0.0001$ ). Platelet-activating factor also caused increased production of superoxide anions

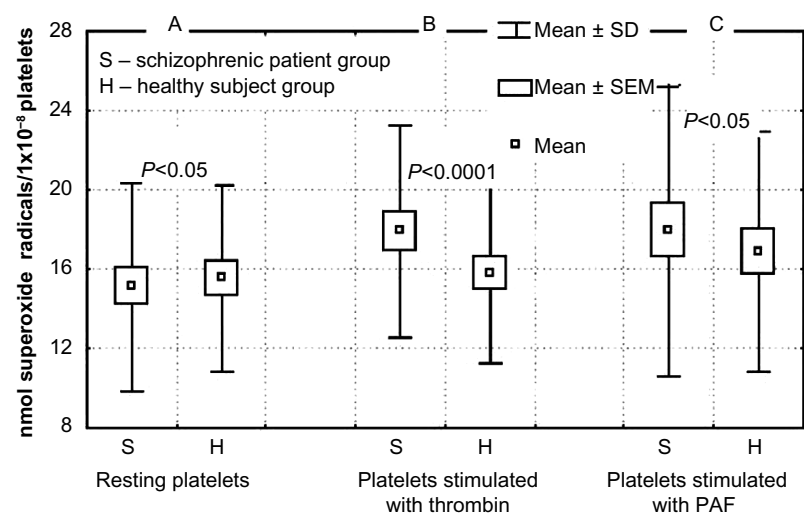

Figure I (A-C) The effects of thrombin $(0.4 \mathrm{U} / \mathrm{mL})$ and platelet-activating factor (PAF; $\left.\mathrm{I} \times 10^{-8} \mathrm{M}\right)$ on the level of superoxide radicals in blood platelets isolated from healthy subjects and from schizophrenic schizophrenic patients $(n=30)$. (A) Levels of superoxide radicals in resting platelets. (B) Levels of superoxide radicals in platelets after stimulation by thrombin $(0.4 \mathrm{U} / \mathrm{mL})$. (C) Levels of superoxide radicals in platelets after stimulation by PAF. Differences in anion radical generation in resting platelets and after agonist stimulation (thrombin or PAF) between groups calculated by Mann-Whitney $U$ test. Abbreviations: SD, standard deviation; SEM, standard error of mean. 
in platelets from both schizophrenic patients and healthy volunteers (Figure $1 \mathrm{C}, P<0.05$ ). In platelets from patients with schizophrenia, the generation of superoxide radicals after thrombin or PAF stimulation was significantly higher than in resting platelets $(P<0.00001, P<0.001$, respectively, Wilcoxon signed-rank test; Figure 1). Differences in the generation of superoxide anions after thrombin or PAF stimulation of platelets in healthy subjects in comparison to resting platelets were not statistically significant. This indicates that platelets from patients with schizophrenia are more sensitive to agonists and produce more superoxide anions than those from healthy subjects. The activity of GPx in platelets from schizophrenic patients was significantly lower $(P<0.00001)$ than in healthy subjects.

\section{Discussion}

Oxidative stress in schizophrenia ${ }^{7}$ may be responsible for altered platelet function, aggregation, and reactivity. ${ }^{32}$ It has been established that platelets of patients with schizophrenia have the increased ability to generate superoxide radicals in response to such agonists as thrombin or PAF, and they are particularly sensitive to thrombin stimulation (Figure 1). The technique used enabled the measurement of superoxide radical production in platelets from schizophrenic patients.

The increased ROS production in stimulated platelets of patients with schizophrenia suggests the involvement of free radicals in the etiopathogenesis of this disease. After binding of the agonist to specific receptors on the platelet surface, several biochemical reactions occur together with ROS production. These include receptor-mediated activation of phospholipase $\mathrm{A}_{2}$ and $\mathrm{C}$ and formation of diacylglycerol, activation of protein kinase $\mathrm{C}$, formation of inositol-1,4,5trisphosphate, and increased calcium-ion levels in cytosol. The platelet stimulation is accompanied by changes in platelet shape, aggregation, and secretion. ${ }^{33}$ In platelets from patients with schizophrenia, increased activity of phospholipase $A_{2}$ changed phosphoinositide metabolism, and peroxidation of lipids has also been shown. ${ }^{2,3,34}$

Platelet lipid peroxidation is a process that leads to membrane damage and changes in platelet function, with altered platelet reactivity to agonists. The response of platelets from patients with schizophrenia to the action of a strong platelet agonist found in this study could be also associated with disturbances of the biochemical pathways in platelets caused by oxidative stress. Platelet response to the action of thrombin or PAF in schizophrenic patients may depend upon the modulated access of specific platelet surface receptors, which can result from changes in platelet membrane. Walsh et $\mathrm{al}^{35}$ demonstrated that the number of platelet integrin receptors $\left(\alpha_{\mathrm{IIb}} / \beta 3\right)$ involved in platelet aggregation in schizophrenic patients is significantly higher in comparison with the platelets of healthy subjects. ${ }^{35}$ The increased expression of platelet integrin receptors for fibrinogen in schizophrenic patients may be responsible for increased platelet reactivity, which probably contributes to the increased risk of cardiovascular diseases in schizophrenia. ${ }^{36-38}$ The peroxidation of PUFAs in platelet membranes can cause a decrease in eicosanoid concentration. Eicosanoids and their precursors, PUFAs, may be involved not only in the pathophysiology but also in the treatment of schizophrenia. Antipsychotics such as clozapine influence thromboxane and chlorpromazine production, and may therefore influence platelet function and exert therapeutic effects in schizophrenic patients. Changes in the concentration of several PUFAs (mainly arachidonic acid) and phospholipase $\mathrm{A}_{2}$ activity were found in erythrocyte and platelet membranes in patients with schizophrenia. ${ }^{2,34,39}$ There is a growing body of evidence that the generation of free radicals and the changed antioxidant defense may

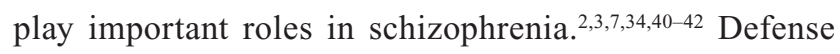
mechanisms against oxidative stress include such antioxidant enzymes as superoxide dismutase (SOD), catalase, or GPx. ${ }^{43}$ SOD activity is decreased in the platelets of patients with schizophrenia. ${ }^{2}$ The results of the present study showed that in platelets from schizophrenic patients, the activity of GPX was significantly lower than in platelets from healthy subjects (controls). This is in agreement with other studies. ${ }^{11,44}$

These data reveal that antioxidative defense mechanisms might be impaired in schizophrenic patients. GPx provides an effective mechanism against cytosolic oxidative injury, because it eliminates hydroperoxide and lipid peroxides by reduction utilizing GSH. The low activity of GPx, which is a selenoenzyme, might be partly dependent on a defect in a selenium-transport protein described in schizophrenia, ${ }^{45}$ on the oxidative/nitrosative alteration of the enzyme, ${ }^{46}$ or suppressed GSH level in platelets when oxidative stress occurs. ${ }^{44,46}$ It should be underlined that in platelets there are two main members of the seleno-GPx family: the cytosolic enzyme cGPx (GPx-1), and phospholipid hydroperoxide GPx (GPx-4), which reduces lipid hydroperoxides in membranes.

Low SOD activity was established in our earlier study, ${ }^{45}$ but this study presents low GPx activity in platelets (Figure 2). In several studies, it has been demonstrated that total antioxidant status of plasma in schizophrenic patients was reduced with impaired antioxidant-enzyme activities in schizophrenia. ${ }^{1,4-6,8,47-50}$ In schizophrenia, a defect in the 


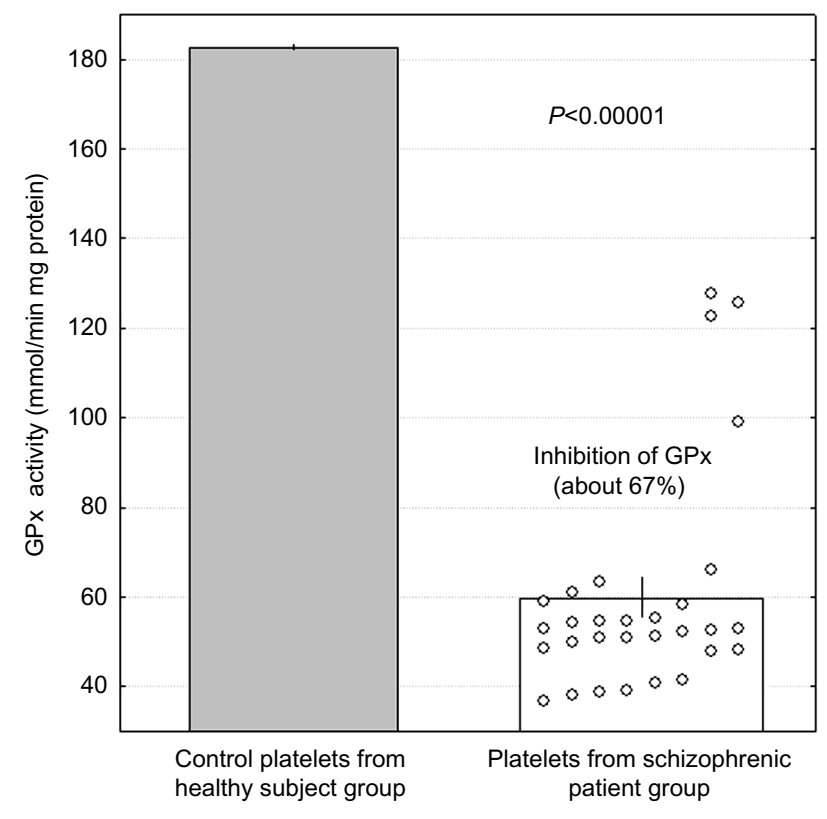

Figure 2 The activity of glutathione peroxidase (GPx) in platelets from patients with schizophrenia $(n=30)$ and healthy subjects (control, $n=30)$. Control group mean 182.5, standard error of mean 0.4 (minimum 178.9, maximum 186.8).

antioxidant defense system under conditions of oxidative stress can lead to oxidative damage to different cells, especially membranes, including blood platelets. ${ }^{19,20}$ In a previous study, it was shown that in patients with schizophrenia, oxidative stress, expressed as the increase of the isoprostane concentration, was elevated. ${ }^{51}$ In platelets of patients with schizophrenia lipid peroxidation, expressed as the concentration of thiobarbituric acid reactive substances, is enhanced, with SOD activity being simultaneously reduced. ${ }^{2}$ The decreased activity of platelet SOD in subjects with psychosis with prominent positive symptoms correlated with the increase of oxidative stress, expressed as the increased level of lipid peroxidation in platelets and production of other ROS. ${ }^{2}$ It appears that the lack of significant increase of superoxide anions generated in resting platelets from patients with schizophrenia presented in this study may be associated with the mechanism that removes the superoxide radicals in the reaction with nitric oxide. ${ }^{19}$ The superoxide anion is a highly reactive radical, and may be decomposed by SOD into $\mathrm{H}_{2} \mathrm{O}_{2}$, or it may produce peroxynitrite as the result of its reaction with nitric oxide (NO) ${ }^{52}$ Peroxynitrite $\left(\mathrm{ONOO}^{-}\right)$ is a highly reactive, short-lived oxidant, which can mediate the oxidation process (nitration and oxidation biomolecules), leading to changes of signal transduction and functioning of platelets. ${ }^{19,20,44,52}$ The role of nitric oxide in schizophrenia has been studied; however, few studies indicate that the generation of nitric oxide in patients with such a diagnosis is increased compared to healthy subjects. ${ }^{8}$ After the reaction of superoxide anion radicals with nitric oxide, the level of superoxide anions is reduced, but lipid peroxidation in platelets can be increased as a result of the reaction initiated also by peroxynitrite. ${ }^{19,44,52}$ The data obtained in this study demonstrate that in platelets from patients with schizophrenia, the generation of superoxide anions after thrombin or PAF stimulation is significantly augmented, suggesting that platelets from schizophrenic patients are more sensitive to platelet agonists. It indicates that activation of platelets in schizophrenia associated with $\mathrm{O}_{2}^{-}$production with a diminished antioxidant defense system may lead to oxidative stress. It seems that the changes in the activities of such peripheral antioxidant enzymes as SOD or GPx in platelets could be considered a biological indicator of severity of the schizophrenia symptoms. ${ }^{6,42,47,48}$

Further research is necessary in order to elucidate the effects of different antipsychotics on antioxidant-enzyme activities and oxidative stress in peripheral cells, such as platelets from schizophrenic patients.

\section{Acknowledgments}

The authors are grateful to Dr Bogdan Kontek from the Department of General Biochemistry, University of Lodz for technical assistance. This work was supported by a grant from the Medical University of Lodz (502/03-1-15502/502-14-109).

\section{Disclosure}

The authors report no conflicts of interest in this work.

\section{References}

1. Ciobica A, Padurariu M, Dobrin I, Stefanescu C, Dobrin R. Oxidative stress in schizophrenia - focusing on the main markers. Psychiatr Danub. 2011;23:237-245.

2. Dietrich-Muszalska A, Olas B, Rabe-Jabłon'ska J. Oxidative stress in blood platelets from schizophrenic patients. Platelets. 2005;16: 386-391.

3. Fendri C, Mechri A, Khiari G, Othman A, Kerkeni A, Gaha L. [Oxidative stress involvement in schizophrenia pathophysiology: a review]. Encephale. 2006;32:244-252. French.

4. Li HC, Chen QZ, Ma Y, Zhou JF. Imbalanced free radicals and antioxidant defense systems in schizophrenia: a comparative study. J Zhejiang Univ Sci B. 2006;7:981-986.

5. Micó JA, Rojas-Corrales MO, Gibert-Rahola J, et al. Reduced antioxidant defense in early onset first-episode psychosis: a case-control study. BMC Psychiatry. 2011;11:26.

6. Raffa M, Atig F, Mhalla A, Kerkeni A, Mechri A. Decreased glutathione levels and impaired antioxidant enzyme activities in drug-naïve firstepisode schizophrenic patients. BMC Psychiatry. 2011;11:124.

7. Reddy R, Yao JK. Free radical pathology in schizophrenia: a review. Prostaglandins Leukot Essent Fatty Acids. 1996;55:33-43.

8. Yao JK, Reddy R. Oxidative stress in schizophrenia: pathogenetic and therapeutic implications. Antioxid Redox Signal. 2011;15: 1999-2002.

9. Yao JK, Reddy R, McElhinny LG, van Kammen DP. Reduced status of plasma total antioxidant capacity in schizophrenia. Schizoph Res. 1998;32:1-8. 
10. Yao JK, Reddy R, van Kammen DP. Oxidative damage and schizophrenia. CNS Drugs. 2001;15:287-210.

11. Berk M, Plein H, Belsham B. The specificity of platelet glutamate receptor supersensitivity in psychotic disorders. Life Sci. 2000;66: 2427-2432.

12. Camacho A, Dimsdale JE. Platelets and psychiatry: lessons learned from old and new studies. Psychosom Med. 2000;62:326-336.

13. Dreux C, Launay JM. [Blood platelets: neuronal model in psychiatric disorders]. Encephale. 1985;11:57-64. French.

14. Plein H, Berk M. The platelet as a peripheral marker in psychiatric illness. Hum Psychopharmacol. 2001;16:229-236.

15. Carotenuto M, Esposito M. Nutraceuticals safety and efficacy in migraine without aura in a population of children affected by neurofibromatosis type I. Neurol Sci. 2013;34:1905-1909.

16. Esposito M, Ruberto M, Pascotto A, Carotenuto M. Nutraceutical preparations in childhood migraine prophylaxis: effects on headache outcomes including disability and behaviour. Neurol Sci. 2012;33: 1365-1368.

17. Iuliano L, Colavita AR, Leo R, Praticò D, Violi F. Oxygen free radicals and platelet activation. Free Radic Biol Med. 1997;22:999-1006.

18. Wachowicz B, Olas B, Zbikowska HM, Buczyn'ski A. Generation of reactive oxygen species in blood platelets. Platelets. 2002;13: 175-182.

19. Olas B, Wachowicz B. Role of reactive nitrogen species in blood platelets functions. Platelets. 2007;18:555-565.

20. Olas B, Nowak P, Kolodziejczyk J, Wachowicz B. The effects of antioxidants on peroxynitrite-induced changes in platelet proteins. Thromb Res. 2004;113:399-406.

21. Do KQ, Trabesinger AH, Kirsten-Krüger M, et al. Schizophrenia: glutathione deficit in cerebrospinal fluid and prefrontal cortex in vivo. Eur J Neurosci. 2000;12:3721-3728.

22. Do KQ, Lauer CJ, Schreiber W, et al. $\gamma$-Glutamylglutamine and taurine concentrations are decreased in the cerebrospinal fluid of drugnaive patients with schizophrenic disorders. J Neurochem. 1995;65: 2652-2662.

23. Berk M, Ng F, Dean O, Dodd S, Bush AI. Glutathione: a novel treatment target in psychiatry. Trends Pharmacol Sci. 2008;29:346-351.

24. Dean O, Giorlando F, Berk M. N-acetylcysteine in psychiatry: current therapeutic evidence and potential mechanisms of action. Psychiatry Neurosci. 2011;36:78-86.

25. Sheehan DV, Lecrubier Y, Sheehan KH, et al. The Mini-International Neuropsychiatric Interview (MINI): the development and validation of a structured diagnostic psychiatric interview for DSM-IV and ICD-10. J Clin Psychiatry. 1998;59:22-33.

26. American Psychiatric Association. Diagnostic and Statistical Manual of Mental Disorders. 4th ed. Washington: American Psychiatric Press; 1994.

27. Wachowicz B, Kustron J. Effect of cisplatin on lipid peroxidation in pig blood platelets. Cytobios. 1992;70:41-47.

28. Jahn B, Hänsch GM. Oxygen radical generation in human platelets: dependence on 12-lipoxygenase activity and on the glutathione cycle. Int Arch Allergy Appl Immunol. 1990;93:73-79.

29. Walkowiak B, Michalak E, Koziolkiewicz W, Cierniewski CS. Rapid photometric method for estimation of platelet count in blood plasma or platelet suspension. Thromb Res. 1989;56:763-776.

30. Paglia DE, Valentine WN. Studies on the quantitative and qualitative characterization of erythrocyte glutathione peroxidase. J Lab Clin Med. 1967;70:158-169.

31. Bradfort MM. A rapid and sensitive method for the quantitation of microgram quantities of protein utilizing the principle of protein-dye binding. Anal Biochem. 1976;72:248-254.
32. Dietrich-Muszalska A, Olas B. The changes of aggregability of blood platelets in schizophrenia. World J Biol Psychiatry. 2009;10: 171-176.

33. Lapetina EG. The signal transduction induced by thrombin in human platelets. FEBS Lett. 1990;268:400-404.

34. Rípová D, Strunecká A, Platilová V, Höschl C. Phosphoinositide signalling system in platelets of schizophrenic patients and the effect of neuroleptic therapy. Prostaglandins Leukot Essent Fatty Acids. 1999;61:125-129.

35. Walsh MT, Ryan M, Hillmann A, et al. Elevated expression of integrin alpha(IIb), beta(IIIa) in drug-naïve, first-episode schizophrenic patients. Biol Psychiatry. 2002;52:874-879.

36. Allebeck P. Schizophrenia: a life-shortening illness. Schizophr Bull. 1989;15:81-89.

37. Allebeck P, Wistedt B. Mortality in schizophrenia. A ten-year follow-up based on the Stockholm Country inpatient register. Arch Gen Psychiatry. 1986;43:650-653.

38. Ruschena D, Mullen PE, Burgess P, et al. Sudden death in psychiatric patients. Br J Psychiatry. 1998;172:331-336.

39. Ross BM. Phospholipid and eicosanoid signaling disturbances in schizophrenia. Prostaglandins Leukot Essent Fatty Acids. 2003;69, 6:407-412.

40. Kuloglu M, Ustundag B, Atmaca M, Canatan H, Tezcan AE, Cinkilinc N. Lipid peroxidation and antioxidant enzyme levels in patients with schizophrenia and bipolar disorder. Cell Biochem Funct. 2002;20:171-117.

41. Mahadik SP, Mukherjee S. Free radical pathology and antioxidant defence in schizophrenia: a review. Schizoph Res. 1996;19:1-17.

42. Raffa M, Mechri A, Othman LB, Fendri C, Gaha L, Kerkeni A. Decreased glutathione levels and antioxidant enzyme activities in untreated and treated schizophrenic patients. Prog Neuropsychopharmacol Biol Psychiatry. 2009;33:1178-1183.

43. Buckman TD, Kling A, Sutphin MS, Steinberg A, Eiduson S. Platelet glutathione peroxidase and monoamine oxidase activity in schizophrenics with CT scan abnormalities: relation to psychosocial variables. Psychiatry Res. 1990;31:1-14.

44. Nowak P, Olas B, Bald E, Głowacki R, Wachowicz B. Peroxynitriteinduced changes of thiol groups in human blood platelets. Platelets. $2003 ; 14: 375-379$.

45. Berry T. A selenium transport protein model of a sub-type of schizophrenia. Med Hypotheses. 1994;43:409-414.

46. Dietrich-Muszalska A, Olas B. Modification of blood platelet proteins of patients with schizophrenia. Platelets. 2009;20:90-96.

47. Miljevic C, Nikolic M, Nikolic-Kokic A, et al. Lipid status, anti-oxidant enzyme defence and haemoglobin content in the blood of long-term clozapine-treated schizophrenic patients. Prog Neuropsychopharmacol Biol Psychiatry. 2010;34:303-307.

48. Padurariu M, Ciobica A, Dobrin I, Stefanescu C. Evaluation of antioxidant enzymes activities and lipid peroxidation in schizophrenic patients treated with typical and atypical antipsychotics. Neurosci Lett. 2010;479:317-320.

49. Raffa M, Barhoumi S, Atig F, Fendri C, Kerkeni A, Mechri A. Reduced antioxidant defense systems in schizophrenia and bipolar I disorder. Prog Neuropsychopharmacol Biol Psychiatry. 2012;39:371-375.

50. Zhang M, Zhao Z, He L, Wan C. A meta-analysis of oxidative stress markers in schizophrenia. Sci China Life Sci. 2010;53: 112-124.

51. Dietrich-Muszalska A, Olas B. Isoprostanes as indicators of oxidative stress in schizophrenia. World J Biol Psychiatry. 2007;8:1-7.

52. Bartosz G. Peroxynitrite: mediator of the toxic action of nitric oxide. Acta Biochim Pol. 1996;43:645-659. 
Neuropsychiatric Disease and Treatment

Dovepress

\section{Publish your work in this journal}

Neuropsychiatric Disease and Treatment is an international, peerreviewed journal of clinical therapeutics and pharmacology focusing on concise rapid reporting of clinical or pre-clinical studies on a range of neuropsychiatric and neurological disorders. This journa is indexed on PubMed Central, the 'PsycINFO' database and CAS.

The manuscript management system is completely online and includes a very quick and fair peer-review system, which is all easy to use. Visit http://www.dovepress.com/testimonials.php to read real quotes from published authors.

Submit your manuscript here: http://www.dovepress.com/neuropsychiatric-disease-and-treatment-journal 